\title{
Some early results related to electrical impedance of normal and abnormal gastric tissue
}

\author{
A. Keshtkar ${ }^{a, *}$, Z. Salehnia ${ }^{a}$, M.H. Somi ${ }^{\text {b,c }}$, A.T. Eftekharsadat ${ }^{\text {b,c }}$ \\ ${ }^{a}$ Medical Physics Department, Medical Faculty, Tabriz University of Medical Sciences, Tabriz, Iran \\ ${ }^{\mathrm{b}}$ Internal Medicine Department, Medical Faculty, Tabriz University of Medical Sciences, Tabriz, Iran \\ ' Liver and Gastrointestinal Diseases Research Centre, Tabriz University of Medical Sciences, Tabriz, Iran
}

Received 4 July 2010; received in revised form 22 December 2010; accepted 23 January 2011

Available online 22 February 2011

\section{KEYWORDS \\ Gastric cancer; \\ Electrical impedance spectroscopy; Minimally invasive technique}

\begin{abstract}
Gastric cancer is the fourth most common cancer and most patients with gastric cancer are being diagnosed in advanced stages of the disease so they do not gain any survival chance from conventional surgical, chemotherapeutic or radiotherapeutic methods. These are relatively high cost procedures in terms of both time and money. This study considers the introduction of a novel minimally invasive diagnostic technique which shows the relationship between histopathology and the electrical impedance spectrum in the human stomach. In this study, 4 electrode technique was used to differentiate tissues from each other using Tabriz Mark 1 electrical impedance system (30 different frequencies in the range of $2 \mathrm{kHz}$ to $1 \mathrm{MHz}$ ). A total of 97 points from 45 patients were studied in terms of their biopsy reports matching to the electrical impedance measurements (in vivo). After impedance measurements and applying calibration factors, a non-parametric statistical technique, the Kruskal-Wallis test was used to evaluate the difference among the groups. According to the calculation of respective data using this spectroscopy system, the resistivity of the normal group was higher than that of the benign group, and the resistivity of these groups were higher than that of the malignant group at frequencies between $470 \mathrm{kHz}$ and $1 \mathrm{MHz}(P<0.05)$. In these frequencies, the impedivity of the dysplastic tissue was significantly lower than that of the other groups $(P<0.05)$. Also, Cole equation fitting procedure was used to generate a scatter plot of the malignant and benign points: it shows in general, benign points had higher values of $R$ than the malignant points. Therefore, electrical impedance spectroscopy can be a useful technique to characterize the stomach tissue.

(c) 2011 Associazione Italiana di Fisica Medica. Published by Elsevier Ltd. All rights reserved.
\end{abstract}

\footnotetext{
* Corresponding author.

E-mail address: mpp98ak@hotmail.com (A. Keshtkar).
} 


\section{Introduction}

Gastric cancer is the fourth most common cancer and second leading cause of cancer related death in the world. According to a global estimation, more than 930,000 new cases of gastric cancer are being diagnosed each year and a minimum of 700,000 patients die from the disease [14]. Unfortunately, the symptoms of early gastric cancer are non-specific and rather vague $[1,4]$. Most patients with gastric cancer are being diagnosed in advanced stages of the disease and they do not have survival chance from conventional surgical, chemotherapeutic or radiotherapeutic methods. Therefore early cancer detection using an efficient surveillance program is a justified way to reduce gastric cancer mortality [13]. These methods of population screening for gastric cancer are being adopted in Japan, South Korea and a part of Taiwan [11]. Various investigations are currently available to help with the diagnosis of gastric cancer as follows: radiological (double-contrast barium) techniques, endoscopy and pathologic assessment, ultrasound scans, CT-Scan, Laparoscopy, and biological serologic tests for serum pepsinogen (PG I, PG II) [4]. Gastric pathology is usually investigated visually by endoscopy. Abnormal areas are usually observed but this can represent a number of conditions ranging from gastritis to adenocarcinoma. Biopsies must be taken from the suspected area in order to obtain diagnostic information. The selection of biopsy sites depends on simple visual inspection so it is effectively random. This is an invasive and relatively high cost procedure in terms of both time and money and is associated with discomfort for the patient and morbidity. It is important to emphasize that annual screening endoscopy and biopsy should be considered for patients who have been found to have atrophic gastritis, dysplasia and adenomatous polyps [1]. Although, the weakness of the above methods are lack of early detection of flat lesions within the stomach. However, the diagnostic accuracy of endoscopy and biopsy for primary upper gastrointestinal cancer is in the 95\% range [4]. Thus, pathologic assessment remains the gold-standard for investigating these patients. In the light of this background, it is possible that electrical impedance spectroscopy may be appropriate for the early detection of flat lesions and assessing gastric pathology. The electrical impedance technique has the ability to characterize any electrical activities between pathologic areas as flat lesion. Thus, this technique can distinguish the pathologic areas from the normal tissue. However, the electrical impedance of normal and malignant regions of any tissue can be different [8].

Therefore, this paper considers the introduction of a novel minimally invasive diagnostic technique to separate pathologic tissue from normal tissue (as some early results related to electrical bio-impedance of normal and abnormal gastric tissue). One example is the relation between tissue structures and electrical flow in cervical neoplasia, to compare the impedance of normal and abnormal cervical tissues [3]. Another study investigated virtual biopsies in Barrett's oesophagus, using electrical impedance measurements [5]. The aim of their study was to show the possibility of differentiating two types of epithelium (squamous and columnar) in terms of their electrical impedances. Another study has been proposed in which electrical impedance spectroscopy can detect bladder pathology, using low and high frequencies $[6,7]$.

\section{Methods}

A total of 97 points from 45 patients were randomly studied using their biopsy reports and the electrical impedance measurements (in vivo). Although, a total of 140 impedance data were recorded from 55 patients, only 97 data from 45 patients were used in this study because they fitted in Cole-Cole equation. The two positive and negative groups which were used to analysis the ROC are the following:

Group 1: benign or non malignant: chronic gastric area and intestinal metaplasia

Group 2: malignant: adenocarcinoma and dysplastic area

Table 1 shows the number of cancerous and benign patients:

According to Table 1, 8 patients had gastric cancer which the impedance data were taken from their normal and pathologic tissues. Later, these were categorized due to their pathological results. The malignant group was patients with Adenocarcinoma (gastric cancer). Bio-impedance measurements were performed at 30 different frequencies in the frequency range of $2 \mathrm{KHz}$ to $1 \mathrm{MHz}$ using electrical impedance spectroscopy system (Tabriz Mark 1). The applied current to measure the transferred impedance in this study was $10 \mu \mathrm{A}$ peak-to-peak which was passing through two electrodes of a small sized probe. This system is a type of research instrument that has been used to characterize the gastric epithelium.

It was designed and constructed in the Electronic Engineering Department, University of Tabriz, Tabriz, Iran. The measured data was transferred to a laptop and picoscope (PC Oscilloscope) using measurement hardware. The resulting potential was measured using the other two electrodes to obtain the electrical impedance of the gastric tissue. The design, construction, development and mechanical assembly of a smaller size probe ( $2 \mathrm{~mm}$ diameter) including 4 gold electrodes to use 4-electrode technique in the gastric tissue were made carefully. The reason of the high importance is because injection of electrical current into living tissue, in vivo and measuring its bio-impedance need a very small sized probe. The size of the probe was $2 \mathrm{~mm}$ total in diameter and sufficient enough to insert into the endoscope (refer to Fig. 1c for details). However the maximum permitted diameter of probe had to be $2 \mathrm{~mm}$ in order to pass through the endoscope to measure the impedance of the inside of gastric tissue. In terms of tip designing, the circular shape of the tip, instead of the planar shape, made the

Table 1 The number of patients and prepared biopsies from every type of tissue.

\begin{tabular}{lll}
\hline Number of patients & Number of biopsies & Tissue type \\
\hline 10 & 22 & Normal \\
8 & 19 & Adenocarcinoma \\
3 & 7 & Dysplastic area \\
24 & 51 & Benign area \\
\hline
\end{tabular}




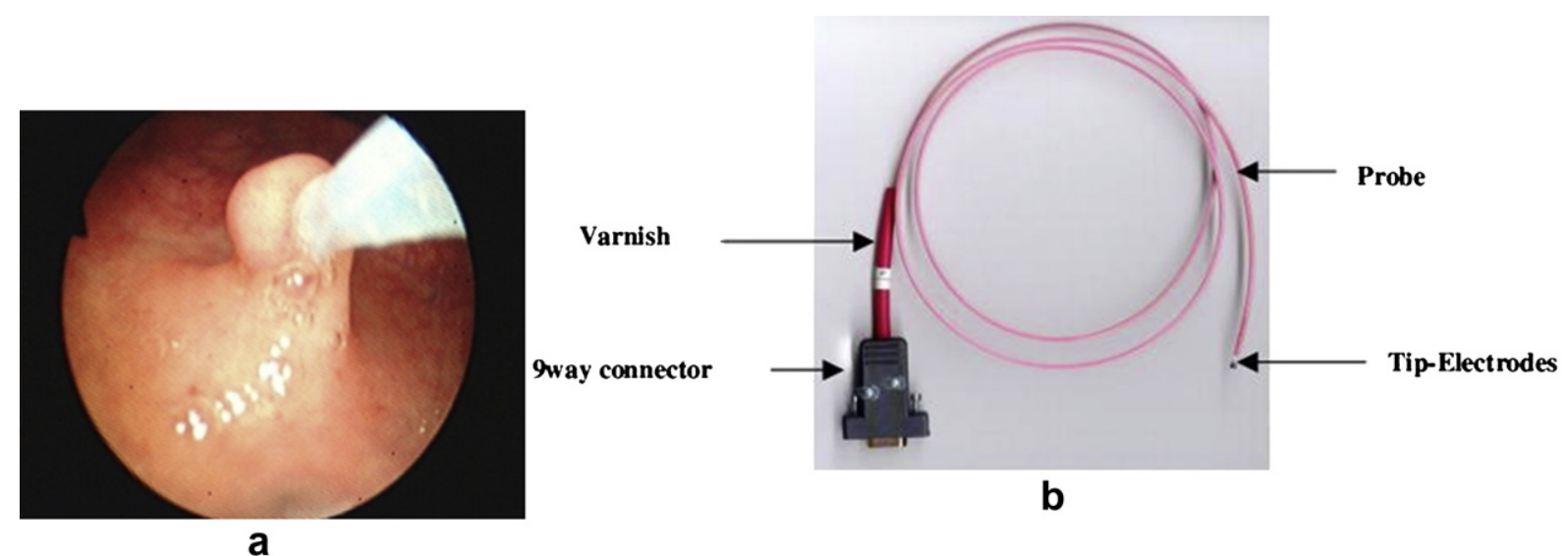

a

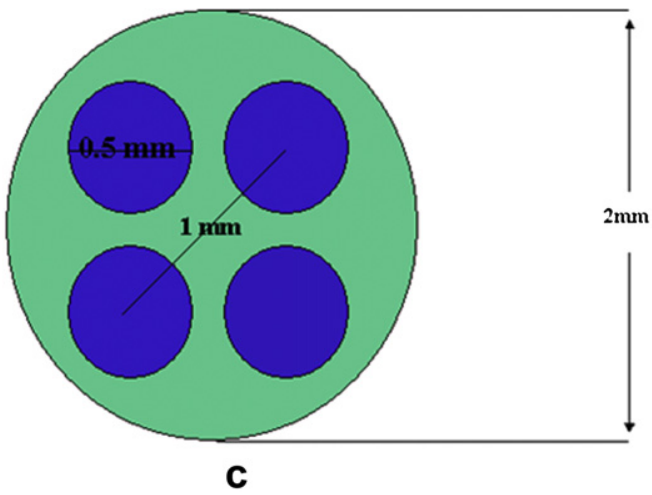

Figure 1 (a) The impedance measurement of gastric tissue. (b) and (c) 4 electrode probe (2 mm in diameter).

tightest tip (smallest configuration) for the probe and thus this shape was chosen. To construct the probe, one can use very accurate tools such as sub-miniature coaxial cable, very small gold wires, small cable stripper and microscope (for electrode-cable soldering) and significant experience and accurate concentration are needed.

To explain this probe in detail, the electrodes are constructed of four gold wire electrodes, $0.5 \mathrm{~mm}$ in diameter, spaced on a $1.6 \mathrm{~mm}$ diameter circle. Total diameter of the probe was $2 \mathrm{~mm}$ and can pass inside of the upper gastrointestinal tract during the endoscopy. More information about the structure of probe can be found in Ref. [6]. Furthermore, the current path is limited to the tissue volume of $2 \mathrm{~mm}$ in diameter and according to our studies for urinary bladder tissue, this area is suitable to consider the tissue impedance, refer to Refs. [15] and [9]. The probe was regularly calibrated using known conductivity of saline solutions by a good conductivity meter before any measurement was taken, to have the tissue impedivity in $\Omega \mathrm{m}$ (it is clear that impedance and resistance has units Ohm and impedivity has $\Omega$ m unit), so what is measured in this study is transfer impedance which is highly dependant on electrode size, geometry and a number of parameters of the tissue and its environment.

The measured impedance data was recorded in a laptop for further data analysis. Also, before any impedance measurement, the probe was sterilized about $30 \mathrm{~min}$. The sterilization procedure was similar to the procedure that is commonly used to clean medical equipments using a special liquid, sidex. After obtaining each impedance data, a biopsy was taken from the measurement area and then fixed separately in formalin solution for histopathological examination (to compare the measured data with the pathological results). See Fig. 1 for details. The applied pressure over the probe and the current path volume was controlled using the first visible indentation (first recordable reading) on the laptop monitor. However, an excessive amount of the applied pressure beyond the first visible indentation (first recordable reading) pressure has a significant effect on the impedance of the living tissue refer to Ref. [10]. Every patient who participated in this study was asked to fill and sign a questionnaire. In addition, Ethics approval was obtained from the Tabriz Medical Ethics Committee, reference number 5/4/1887.

According to the reports, changes of the gastric epithelium were classified histologically into normal mucosa, benign changes (chronic gastritis and intestinal metaplasia), dysplasia, and tumoral groups. In this work, ' Straight Line Fitting Program' was used to fit this model to the measured impedance data. As it is clear, the spectrum of the electrical impedance of the gastric tissue is in different frequencies, and is suitable to distinguish different tissues. Biological tissue have complex electrical impedance in different frequencies, as it was used in the real part of this impedance (in $\Omega \mathrm{m}$ ) because its SNR (signal to noise ratio) is more than the imaginary part [12]. Sometimes, authors use half circle arcs (i.e., complex plane locus) to show the electrical impedance of living tissues in different frequencies. However, we did not use these curves in our studies and we were interested to show the impedance differences using impedance against frequency curves (in several published papers).

Furthermore, to evaluate the significance of separating measured impedance spectrum, a non-parametric statistical test, the Kruskal-Wallis test, was applied to these data (the impedance data were not distributed normally 
due to the box-plots resulting from the measured data). Finally, the ROC curves for the parameters R and R/S was applied to the measured data to evaluate the possibility of individual classification of the benign and malignant points.

It must be mentioned that in order to analyze data, a simple tissue equivalent model, the cole equation model, was used in this study. This model considers the tissue as a combination of two resistors and a capacitance: one resistor $(R)$, the extra-cellular space is in parallel with a series of a membrane capacitance $(C)$, and another resistor(S), the intra-cellular space [6]. $\mathrm{R}, \mathrm{S}$ and $\mathrm{C}$ were used as input data to fit ROC curve in this study. These parameters have an important role to distinguish malignant areas from the normal parts of the living tissue.

\section{Results}

Calibration factors were calculated and averaged for each frequency using simple M-file written in Matlab program. By considering these factors, impedivity were calculated for normal, benign, malignant and dysplastic areas of the gastric tissue (with considering their pathological reports). Then, after applying suitable M.files in Matlab media, the relationship between the tissue impedance and frequency at $470 \mathrm{kHz}-1 \mathrm{MHz}$ for normal, benign, malignant and dysplastic areas is shown in Fig. 2. We were interested to measure impedance data in wide range of frequency, $2 \mathrm{kHz}$ to $1 \mathrm{MHz}$. However, because of unwanted noises in lower frequencies, it was impossible to have all the data which can be fitted in the cole equation or straight line programme. Thus, we were forced to ignore the lower frequency results and tried to plot only the data for $470 \mathrm{kHz}$ to $1 \mathrm{MHz}$ frequencies range. The circular, rectangular, triangular and diamond shape points show the mean value of the real part of impedivity for every point in different frequencies (in $\Omega \mathrm{m}$ ).

These are related to normal, benign, malignant and dysplastic areas, respectively. It is clear that the overlapping of the groups will preclude the classification of

b

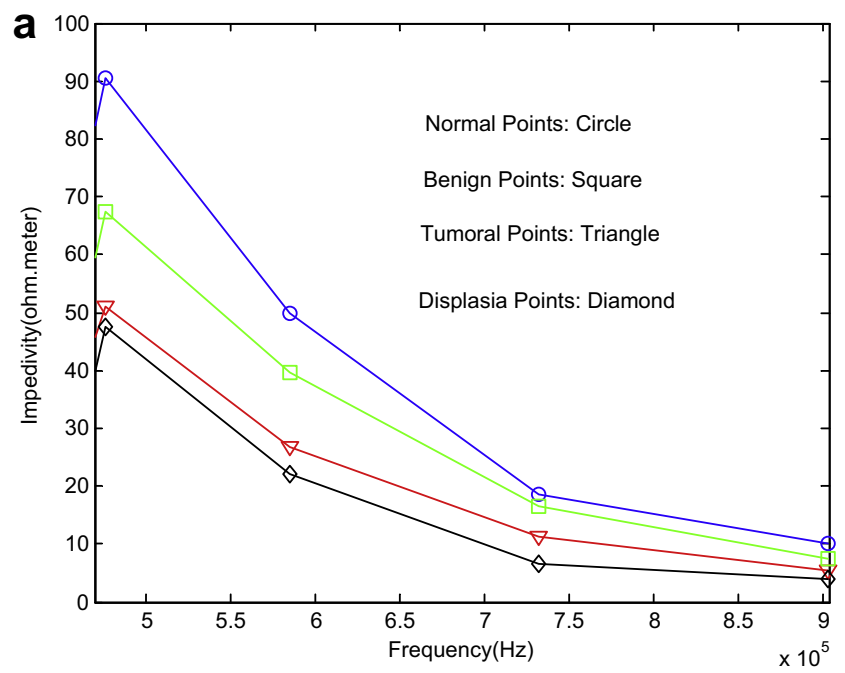

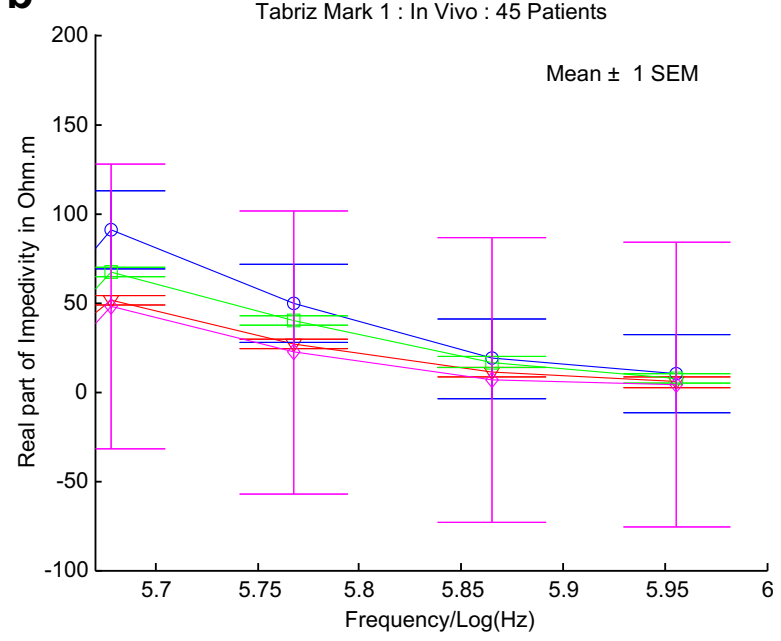

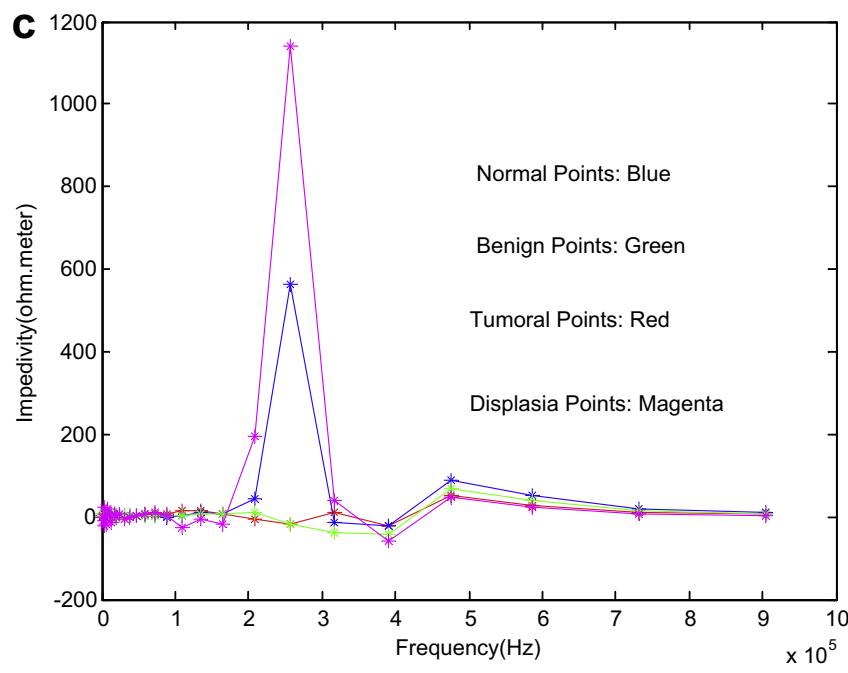

Figure 2 Real part of impedivity versus frequency for normal, benign, malignant and dysplastic areas of the gastric tissue (the error bars demonstrate the standard error of the mean). (a) Shows a linear relationship of impedance against frequency and (b) demonstrated this relationship in logarithmic scales in frequency range of $470 \mathrm{kHz}$ to $1 \mathrm{MHz}$ (c) shows the measured frequency range of $2 \mathrm{kHz}$ to $1 \mathrm{MHz}$. 
individual measurements. A non-parametric statistical technique, the Kruskal-Wallis test was used to evaluate the difference among the groups. According to the calculation of respective data, the resistivity of normal group was higher than that of the benign group, and the resistivity of these groups was higher than that of the malignant group at frequencies between $470 \mathrm{kHz}$ and $1 \mathrm{MHz}(P<0.05)$. In these frequencies, the impedivity of dysplastic tissue was significantly lower than that for other groups $(P<0.05)$.

There is a plot that resulted from applying Straight Line Fitting Program to the measured data. There were 26 malignant and 47 benign points. However, a total of 97 points were measured and only 73 points $(26+47)$ were used in ROC analysis because these points were fitted in Straight Line Fitting Program. These points demonstrated a group separation between two malignant and benign groups from each other (Fig. 3). The fitting software (Matlab-M.file) that used in this study to fit the data is 'Straight Line Fitting Programme'. The parameters $\mathrm{R}, \mathrm{S}$, and $\mathrm{C}$ were used as fitting parameters. The real part of impedivity was used to separate malignant points from the benign area of the gastric epithelium. It means that due to the determination of extra-cellular and intra-cellular impedivities from the Cole equation model, the malignant and benign points are placed on the left-hand and right-hand sides of Fig. 3, respectively.

The scatter plot suggests that classification of malignant areas may not be possible, because the false-negative and false-positive rate may be high. Therefore, this has been studied using the receiver operating characteristic (ROC) curves for the parameters $R$ and R/S separately. These parameters are shown in Fig. 4.

\section{Discussion}

According to the studies carried out by Brown et al. in 1998 on the cervical tissue [2] and Gonzales-Correa et al. in 1999 on the oesophagus tissue [5], the impedivity of malignant

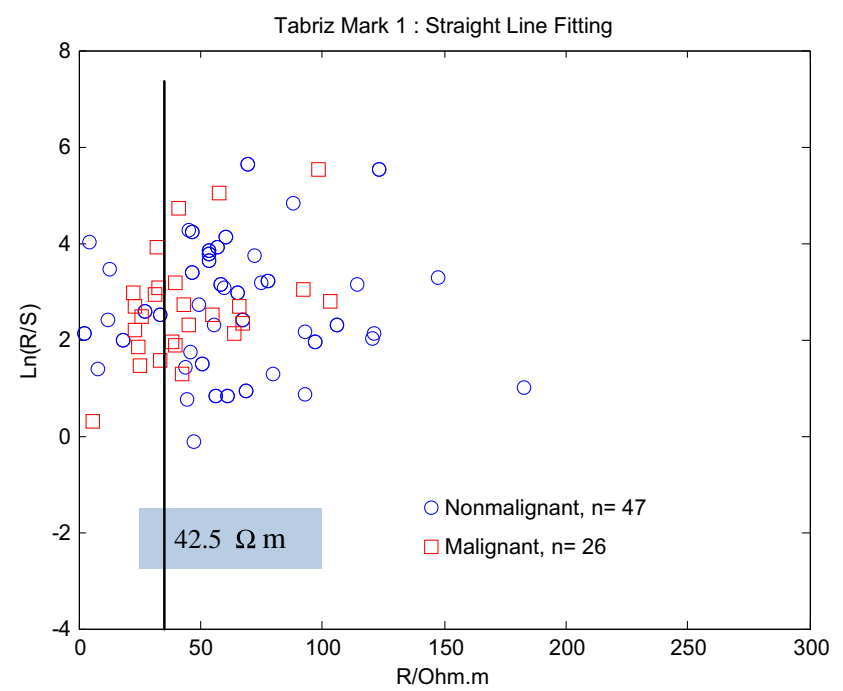

Figure 3 The straight line fitting for benign and malignant areas of the gastric tissue. ( $R$ is impedivity of gastric tissue in $\Omega \mathrm{m}$.
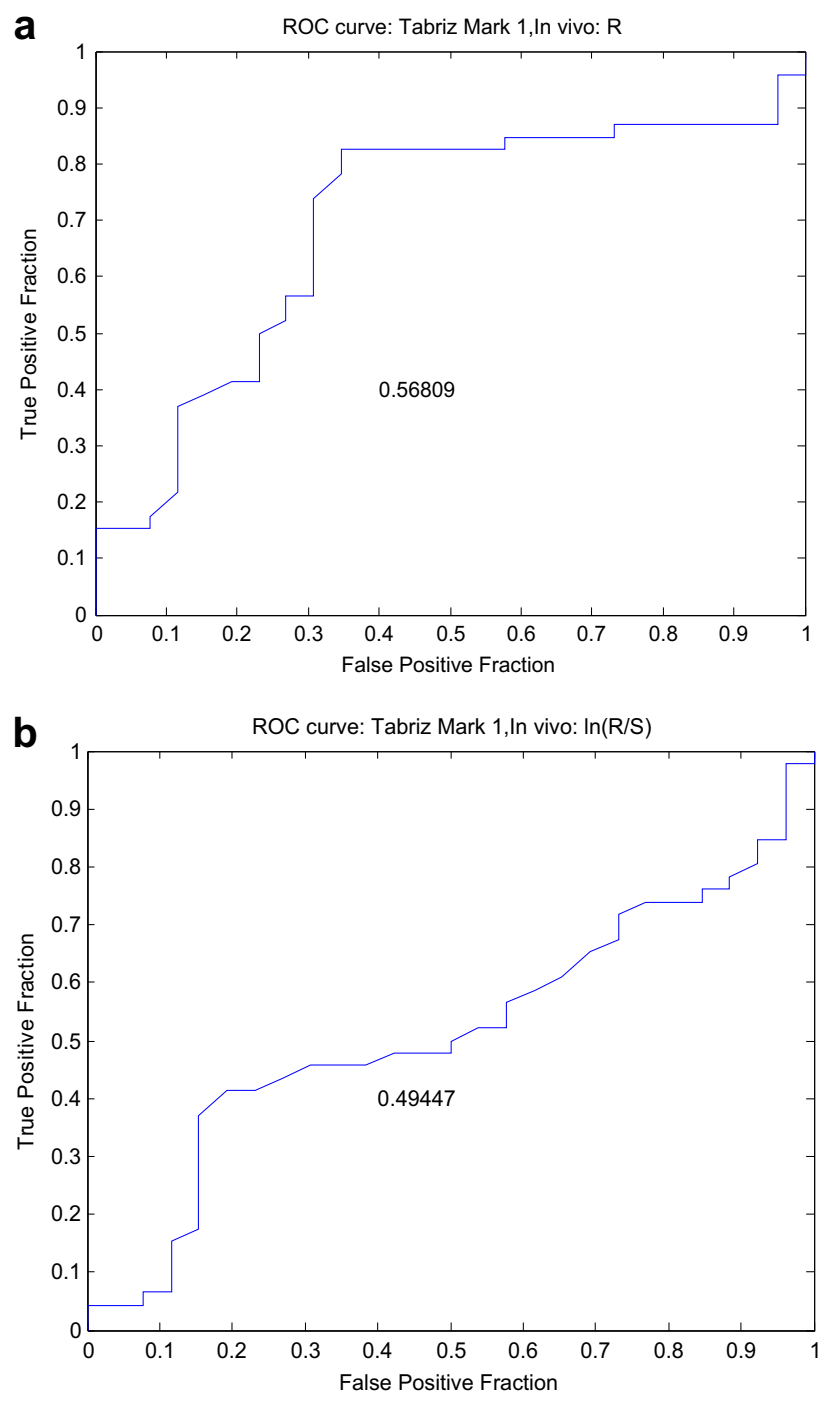

Figure 4 The ROC curves for the parameters R and R/S: (a) R and (b) R/S.

tissue was significantly lower than of the normal tissue $(9.6-614 \mathrm{kHz})$. However, in this study, at $470 \mathrm{KHz}-1 \mathrm{MHz}$, the impedivity of malignant area of the stomach tissue is significantly lower than that of the benign and normal areas (Fig. 2). These results of the gastric tissue are in agreement with the above-mentioned results. Also, according to the fitting model, the malignant and benign points are placed (approximately) on the left-hand and right-hand sides of Fig. 3, respectively. However, there is only a grouped data separation and not a perfect categorization of the individual measurements. Moreover, to distinguish the individual data, which are not placed in its groups, one can consider the receiver operating characteristic (ROC) curves for the parameters. The ROC curve for measurements indicates that the technique could provide diagnostic information partly. An important provision is that the data relate to individual biopsies, and not to individual patients, and the aim is to identify patients with malignancy that requires further investigation. Therefore, in vivo results suggest that it not possible to diagnose patients with 
malignancy using electrical impedance spectroscopy now and we need more study to improve our results.

Finally, an important message of this paper can be the separation ability to distinguish malignancy of the stomach tissue. Thus, this minimally invasive technique would assist the gastroenterologist to detect appropriate sites for biopsy because current diagnostic methods use random biopsies. Therefore, this technique can be a complementary method for endoscopy, biopsy and histopathological evaluation of the stomach abnormalities.

Figure 4 indicates that R/S is a lousy parameter (random result), and an area under curve of 0.57 for the R parameter is not very encouraging, thus, two ROC curves did not show a good separation between malignant and benign groups. However, $\mathrm{R}, \mathrm{S}$ and $\mathrm{C}$ are the main electrical characteristics parameters of the living tissue and they are used for this purpose $[5,8]$. Therefore, the surface area under these curves demonstrated a low number and shows a weak diagnostic technique. However, this may be because of the small size of the probe ( $2 \mathrm{~mm}$ in diameter) or our new impedance spectroscopy system. This problem can be overcome by considering the effect of gastric liquids on the measured impedance and using ex vivo studies by bigger probe and so on. This system was used for the first time to measure impedance in vivo for human living tissue. For improving our system and further studies, it is needed to work hard and improve this minimally invasive technique in future. Thus, after improvements, it can be a good screening technique for gastric caner, bladder cancer, cervical cancer and breast cancer. Therefore, according to our results from ROC curves, it is difficult to introduce this technique to have a high specificity level (i.e., $90 \%$ specificity) to show the performance of this new technology now.

\section{Conclusion}

The electrical impedance data of the gastric epithelium were measured and compared with histopathological reports of the biopsies, which had been taken from the measurement area. The final results show that the resistivity of the normal group was higher than that of the benign and the resistivity of these groups was higher than that of the malignant group at frequencies between $470 \mathrm{kHz}$ and $1 \mathrm{MHz}(P<0.05)$. In these frequencies, the impedivity of dysplastic tissue was significantly lower than that of the other groups $(P<0.05)$. These results of the gastric tissue are in agreement with the results obtained from the oesophagus and the cervical tissues. The straight line fitting programme was used to generate a scatter plot of the malignant and benign points: in general, benign points had higher values of $\mathrm{R}$ than the malignant points. Moreover, the overlapping of the groups will preclude the classification of individual measurements. To evaluate individual points which were not placed in their group, ROC curves were used. These curves for the impedance data indicated that this technique could provide diagnostic information partly and the aim is to identify patients with malignancy that requires further investigation. According to the analyzed measured data and the results shown in the figures, the impedivity of the malignant gastric tissue is significantly lower than the impedivity of the benign and normal tissues. Finally, if the noise problem could be better controlled, more information could be extracted from the spectra and then provide more power to the classification.

\section{Acknowledgments}

This study was extracted from an MSc thesis (6/7-2/87) and supported financially by Liver and Gastrointestinal Diseases Research Centre, Tabriz University of Medical Sciences, Tabriz, Iran. We thank Dr. M H Somi and his colleges for their support and assistance with this project.

\section{References}

[1] Barr H. Gastric tumours. Medicine 2007;35(4):216-9.

[2] Brown BH, Tidy JA, Boston K, Blackett AD, Sharp F. Tetrapolar measurement of cervical tissue structure using impedance spectroscopy. In: IEEE Conference on Biomedical Engineering. Hong Kong; 1998.

[3] Brown BH, Tidy JA, Boston K, Blackett AD, Smallwood RH, Sharp F. Relation between tissue structure and imposed electrical current flow in cervical neoplasia. The Lancet 2000; 355:892-5.

[4] Catalano V, Labianca R, Beretta GD, Gatta G, De Braud F, Van Cutsem E. Gastric cancer. Critical Review in Oncology Hematology 2005;54:209-41.

[5] Gonzales-Correa CA, Brown BH, Smallwood RH, Kalia N, Stoddard CJ, Stephenson TJ, et al. Virtual biopsies in Barrett's oesophagus using an impedance probe. Annals of the New York Academy of Sciences 1999;873:313-21.

[8] Keshtkar A, Smallwood RH. Electrical impedance spectroscopy and diagnosis of bladder pathology. Physiological Measurement 2006;27:585-96.

[7] Keshtkar A. Virtual bladder biopsy using bio-impedance spectroscopy at $62.500 \mathrm{~Hz}-1.024 \mathrm{MHz}$. Measurement 2007;40: 585-90.

[6] Keshtkar A. Design and construction of small sized pencil probe to measure bio-impedance. Medical Engineering \& Physics 2006;29:1043-8.

[9] Keshtkar A. 'Modelled current distribution inside the normal and malignant human urothelium using Finite Element Analysis'. IEEE Transactions on Biomedical Engineering February 2008;55(2).

[10] Keshtkar A. 'The effect of applied pressure on the electrical impedance of the bladder tissue using small and large probes'. Medical Engineering and Technology November-December 2008;32(6):505-11.

[11] Leung WK, Wu MS, Kakugawa Y, Kim JJ, Yeoh KG, Goh KL, et al. Screening for gastric cancer in Asia: current evidence and practice. Lancet Oncology 2008;9:279-87.

[12] Lu L, Hamzaoui L, Brown BH, Rigaud B, Smallwood RH, Barber DC, et al. "Parametric modelling for electrical impedance spectroscopy system." Medical \& Biological Engineering \& Computing 1996;34(2):122-6.

[13] Malekzadeh R, Derakhshan MH, Malekzadeh Z. Gastric cancer in Iran: epidemiology and risk factors. Archives of Iranian Medicine 2009;12(6):576-83.

[14] Parkin D, Bray F, Ferlay J, Pisani P. Global cancer statistics, 2002. CA: A Cancer Journal for Clinicians 2005;55:74-108.

[15] Walker DC, Smallwood RH, Keshtkar A, Wilkinson BA, Hamdy FC, Lee JA. Modelling the electrical properties of bladder tissue-quantifying impedance changes due to inflammation and oedema. Physiological Measurement 2005;26: 251-68. 